\title{
Role of Preimplantation Genetic Diagnosis (PGD) in Current Infertility Practice
}

\author{
Helen Ghislaine Tempest, Joe Leigh Simpson \\ Florida International University Herbert Wertheim College of Medicine, Miami, Florida, USA
}

Correspondence: Joe Leigh Simpson, Florida International University Herbert Wertheim College of Medicine, 11200 SW 8th St. Miami, FL 33199, USA, e-mail: simpsonj@fiu.edu

\section{ABSTRACT}

Chromosome imbalances are the leading cause of pregnancy loss in humans and play major roles in male and female infertility. Within the past two decades, the development and application of preimplantation genetic diagnosis (PGD) has played an important role in infertility practices worldwide. The purpose of this review is to discuss, how PGD may be applied in combating numerical chromosomal abnormalities and in Robertsonian and reciprocal chromosome translocations. We shall consider prevalence and risk of each aberration, interchromosomal effects and rationale behind use of PGD in each case. Numerical chromosome abnormalities (aneuploidy and polyploidy) in particular affect a very high proportion of preimplantation embryos ( 50\%). Given that a majority of preimplantation embryos are aneuploid, PGD can be used to screen embryos and transfer euploid embryos to improve pregnancy rates and reduce spontaneous abortions. The rationale of utilize PGD to transfer only euploid embryos would seem sound, but controversies exist surrounding application of PGD for aneuploidy detection. To this end, we will discuss the dichotomy between favorable descriptive reports and less favorable randomized clinical trial data. This review will discuss the trend towards differing sources of embryonic DNA (e.g. polar body vs blastomere vs blastocyst) as well as development of novel technologies for 24 chromosomes analysis.

Keywords: Preimplantation genetic diagnosis (PGD), Infertility, Aneuploidy.

\section{INTRODUCTION}

\section{Infertility-Subfertility}

Preimplantation genetic diagnosis (PGD) can play important but specific roles in infertility treatment. One applicable approach arises in parents having a predisposition for chromosomally abnormal conceptions. An obvious example is presence of a balanced translocation in one partner. However, predisposition is often subtle and difficult to prove, as in repeated pregnancy losses of unknown etiology. The second general approach applies to couples with no ostensible genetic predisposition. Would they improve their likelihood of success through assisted reproductive technologies (ART) by transfer of only euploid embryos? PGD and transfer of chromosomally normal (euploid) embryos should ameliorate the universally low ART success rate, which is typically $30 \%$ even with transfer of $>1$ embryo. Does this occur?

In this contribution, unavoidably reflecting other recent publications, ${ }^{1,2}$ we shall explore these two general approaches toward using PGD in the treatment of infertility.

\section{Chromosomal Translocations Prevalence and Risk}

A balanced translocation is found in 3 to $5 \%$ of couples experiencing repeated pregnancy losses. These individuals are

Date of Received: 06-09-10

Date of Acceptance: 01-10-10

Date of Publication: Sep. 2010 themselves phenotypically normal, but their offspring (abortuses or abnormal liveborns) may be show chromosomal duplications or deficiencies as result of normal meiotic segregation of the balanced translocation.

Structural rearrangements can give rise to unbalanced gametes. The relative frequency of normal or unbalanced oocytes or sperm depends on the chromosomes involved, size of the involved segments, presence of heterochromatin, tendency for recombinational events, and breakpoints locations (G-positive or G-negative bands). ${ }^{3}$ The great majority of segregants are unbalanced and often lethal. The exact frequency of unbalanced gametes in a given individual varies widely, presumably reflecting the specific rearrangement involved. In addition to the theoretical predictions for unbalanced with straight forward segregation, other untoward events also occur (e.g. 3:1 rather than 2:2 segregation).

The likelihood of a female patient having a balanced translocation changes relatively little following one, two, three or four miscarriages: $0.8 \%, 1.7 \%, 2.3 \%$, and $2.9 \%$, respectively. ${ }^{4}$ For males, the respective rates are $1.2 \%, 1.9 \%$, $2.4 \%$, and $0(0 / 39)$. The frequency of balanced translocations is increased further in the presence of a family history of a stillborn or abnormal liveborn.

\section{Robertsonian Translocations}

Robertsonian translocations involve centric fusion of two acrocentric chromosomes (Nos. 13, 14, 15, 21, 22). The theoretical risk of a parent with a translocation involving nos. 
14 and 21 [t(14q;21q)] having a liveborn child with Down syndrome is $33 \% .^{5}$ Empirical risks are much less, only $2 \%$ if the father carries such a translocation and $10 \%$ if the mother does. The explanation for the very much lower empiric than theoretical risks lies in part, the lethality of most segregant products, and perhaps also due to some form of meiotic drive, toward preferential fertilization involving a normal gamete. Robertsonian (centric fusion) translocations involving chromosomes other than chromosome 21 show even lower empirical risk. In $\mathrm{t}(13 \mathrm{q}: 14 \mathrm{q})$, the risk for liveborn trisomy 13 is $1 \%$ or less. ${ }^{6}$ The above figures reflect liveborns. In theory one could predict these risk rates by studying gametes.

To date, the sperm of over 20 cases of balanced Robertsonian translocations has been analyzed to determine the frequency of unbalanced sperm in male Robertsonian translocation carriers. These studies report between 3 to $30 \%$ unbalanced sperm. ${ }^{7}$ The extent to which empiric counseling should be altered based on proportion of unbalanced sperm is still arguable.

\section{Reciprocal Translocations}

In reciprocal translocations, interchanges occur between two or more chromosomes, but do not involve two acrocentric chromosomes. Empirical data for specific translocations are usually not available, primarily due to reciprocal translocations being unique to individual families. Unlike Robertsonian translocations, the same reciprocal translocation is rarely observed among different families. Each is seemingly unique with the exception of $\mathrm{t}(11 ; 22)$ (q23.3;q11.2) and $\mathrm{t}(4 ; 8)$ (p16;p23) and several translocations associated with various cancers. Generalizations in counseling must then be made on the basis of data pooled from many different translocations. Theoretical risks for abnormal offspring (unbalanced reciprocal translocations) are much greater than empirical risks, but differences between sexes are less apparent. Empiric risks are $12 \%$ for abnormal livebirths in offspring of either female heterozygotes or male heterozygotes. ${ }^{6}$ Among 30 reciprocal translocations in which individual sperm have been studied the percentage of unbalanced spermatozoa is much higher than that in Robertsonian translocations, the reported range of 29 to $81 \%{ }^{7}$ The wide variation of unbalanced sperm among reports, presumably reflects the specific chromosomes, size of segments involved and predisposition for recombination within the given translocated region(s).

\section{Interchromosomal Effect (ICE)}

An increase in frequency of chromosomal abnormalities exists unrelated to the chromosome translocation being investigated. This is termed an "interchromosomal effect" (ICE). That is, abnormal behavior occurs among chromosomes not involved in the structural rearrangement. Thus, individuals with a balanced translocation may be at increased risk for chromosome nondisjunction involving chromosomes not involved in the structural rearrangement. ${ }^{7}$ It appears that an ICE exists in 58\% of Robertsonian translocations and $64 \%$ of reciprocal translocations. ${ }^{8}$ PGD studies have confirmed that embryos of chromosomal translocation carriers have a high frequency of genetic abnormalities. ${ }^{8}$ Studies have also suggested an ICE for Robertsonian translocation carriers, because there appears to be a higher frequency of aneuploidy, ${ }^{9-11}$ however, others have not found a significant increase. ${ }^{12,13}$ Given the association between structural chromosome aberrations and involvement of other chromosomes, it does not seem unreasonable that PGD fluorescent in situ hybridization (FISH) for translocations should not only include translocation chromosomes but also chromosomes X, Y, 13, 18, 21 and 16 to exclude additional chromosome errors.

\section{Clinical Rationale for PGD in Infertility Management}

The likelihood of a normal/balanced carrier livebirth in a couple one of whom has a balanced translocation is 65 to $70 \%$, not different from couples with recurrent pregnancy loss but without a translocation (Table 1). However, there is one important caveat: increased time to achieve pregnancy compared to those couples not having a translocation partner. With a balanced translocation the mean time to achieve pregnancy without PGD is 4 to 6 years. ${ }^{14-16}$ Although this time interval may not be intolerable for younger women, a balanced translocation is alone considered an indication for preimplantation genetic diagnosis (PGD) especially in older women. ${ }^{17}$ In one study of translocation couples the lifetime cumulative pregnancy rate with PGD was $57.6 \%$, with an average of only 1.24 cycles. ${ }^{18}$ This short-time frame for translocation couples undergoing PGD to achieve pregnancy clearly contrasts with the afore mentioned 4 to 6 years in couples, who do not use PGD. For that reason, the society for assisted reproductive technology (SART) guidelines support this indication for PGD. ${ }^{17}$ This advice holds irrespective of whether PGD improves livebirth rate.

PGD for infertile couples having a balanced translocation should preclude not only loss but also an abnormal liveborn. ${ }^{18-20}$ Otani et al ${ }^{18}$ observed only 5.3\% spontaneous abortions after PGD for translocations, far fewer than expected. A caveat is that because relatively few embryos are normal or even balanced, many embryos (e.g. 10-12) are

Table 1: Approximate recurrence risk figures useful for counseling women with repeated spontaneous abortions

\begin{tabular}{ccc}
\hline & Prior abortions & Risk (\%) \\
\hline Women with liveborn infants & 0 & $5-10$ \\
& 1 & $20-25$ \\
Women without liveborn infants & 2 & 25 \\
& 3 & 30 \\
& 4 & 30 \\
& 3 & $30-40$ \\
\hline
\end{tabular}

Prepared by Simpson and Jauniaux ${ }^{96}$ from multiple sources. 
needed to assure transfer of 1 to 2 genetically normal or balanced embryos. Randomized clinical trials (RCTs) demonstrating efficacy with respect to increased livebirths have not been conducted, but descriptive data are nonetheless compelling.

\section{Distinguishing Genetically Normal from Balanced Chromosome Translocations}

A diagnostic caveat is that PGD using FISH is applicable only in interphase analysis. Traditionally, the probes used are commercially available and chromosome specific, but not breakpoint specific. This approach thus cannot distinguish an embryo with a balanced translocation from a genetically normal embryo. Breakpoint specific probes could accomplish this, but would need to be customized due to unique chromosome translocation breakpoints. ${ }^{21}$ This requires lengthy test development time due to unique chromosome translocations, and prohibitive expense.

Several approaches have recently been developed to distinguish genetically normal from balanced gametes. This is predominantly made possible through “conversion” from interphase nuclei to metaphase, thus enabling visualization of chromosomes from single blastomeres. Originally, this was accomplished by fusing human blastomeres with enucleated, intact mouse zygotes or oocytes, ${ }^{22-24}$ followed by fixation of the resultant heterokaryons at the metaphase stage of the first cleavage division. ${ }^{25}$ Although, this method was relatively successful in yielding results, it raised ethical concerns related to the formation of interspecies heterokaryons. Perhaps more importantly, this technique was extraordinarily labor intensive and technically demanding, few laboratories worldwide being able to implement this technology. More recently, a chemical conversion method has been developed for blastomeres, utilizing caffeine and low dose colcemid or electrical stimulation. ${ }^{22-26}$ Although these approaches were originally not always consistently successful, recent experience has been more promising. Kuliev et al. ${ }^{25}$ report that $75.7 \%$ of 1451 blastomeres analyzed by conversion methods yielded diagnostic results, albeit with the addition of FISH methods in $12.7 \%$ of the 1451 . In the $24.3 \%$ of cases in which metaphase conversion failed, standard interphase FISH was still possible in 18.8\%. Overall, a diagnostic result was possible in 95\% of cases (1371 of 1451). These data support the conversion method as a relatively robust and suitable approach for translocations providing rapid results and allowing distinction between normal, balanced and unbalanced embryos in $75.7 \%$ of cases. Another approach for distinguishing genetically normal from balanced gametes involves haplotyping utilizing multiplex fluorescent PCR for highly polymorphic markers. ${ }^{27}$ Microarray technology offers yet another potential approach. However, at present PCR and microarray approaches do not allow for fresh cycle transfers, requiring cryopreservation of embryos and transfer at a subsequent cycle.

\section{Pregnancy Loss Due to Recurrent Aneuploidy}

\section{Frequency of Losses and Spectrum of Chromosomal Abnormalities}

Frequency of pregnancy losses in humans is very high and at no stage is it higher than in preimplantation embryos. In morphologically normal embryos, 25 to 50\% show numerical chromosomal abnormalities (aneuploidy or polyploidy), ${ }^{28}$ increasing with maternal age. The frequency of chromosomal abnormalities in morphologically normal embryos is at least $75 \%$, again principally aneuploidy. These data are based on studies using fluorescence in situ hybridization (FISH) with chromosome specific probes for only seven to nine chromosomes, rates should be higher if either 24 chromosomes FISH or array comparative genome hybridization (CGH) were utilized (these methods are discussed below). The 25 to 50\% aneuploidy rate in morphologically normal embryos is, in turn, consistent with 5 to $10 \%$ aneuploidy in sperm of ostensibly normal males and in 20\% aneuploidy of oocytes of women undergoing ART. ${ }^{29,30}$ Aneuploidy rates in oocytes and embryos predictably increase as maternal age increases.

Trisomy for every chromosome has been observed in preimplantation embryos. The most common trisomies in clinical abortuses are 22, 21, 16, 15, 14 and 13 (in descending order). In preimplantation embryos, these are also the most common trisomies, accounting in aggregate account for 60 to $70 \%$ of trisomies. The frequency of a given trisomy is an important consideration in selecting chromosome specific probes in preimplantation genetic diagnosis (PGD) aneuploidy testing. Testing only the five chromosomes capable of reaching term (X, Y, 13, 18 and 21) only detects 28 to $31 \%$ of aneuploidies, screening additional chromosomes 15, 16, 17 and 22 (nine chromosomes) increases detection to 57 to $72 \%$, adding chromosomes 8, 14 and 20 (12 chromosomes) allows detection of 80 to $91 \%$ of abnormal embryos. ${ }^{31,32}$ (See below concerning double trisomies).

Most trisomies show a maternal age effect, but the effect varies among chromosomes. Increasing maternal age also correlates positively with errors at meiosis I, the most common cytological explanation (95\%) for trisomies. Most of these are meiosis I but the proportion of trisomies arising at meiosis I versus meiosis II varies per chromosome. Virtually all trisomy 16 cases are maternal in origin. Initially, it was thought these arise exclusively at meiosis I, but Kuliev et $\mathrm{al}^{25}$ recently showed meiosis II errors are responsible as well. Moreover, the error in oogenesis often involves a single chromatid rather than the entire chromosome. Errors in paternal meiosis account for $10 \%$ of acrocentric trisomies. In (rare) nonacrocentric trisomies, paternal meiotic errors are equally likely to arise at meiosis I or II.

Double trisomy occurs, most often involving the $\mathrm{X}$ chromosome and an autosome. Maternal age is higher than found in single trisomy. This observation is clinically relevant because rarer trisomies frequently coexist with a more common 
trisomy. Distinguishing normal embryos from abnormal embryos may thus not necessarily require analysis of all 24 chromosomes. Given double trisomies, in fact, testing only 10 to 12 chromosomes identifies 90\% of all numerically abnormal embryos. ${ }^{31}$ That is, it is not necessarily obligatory to detect all specific aneuploidies, if one can distinguish embryos that have and do not have chromosomal abnormalities.

Triploidy accounts for $25 \%$ of chromosomally abnormal abortuses and is typically $69, \mathrm{XXY}$ or $69, \mathrm{XXX}$. The origin is usually dispermy (90\%). Triploidy may follow either fertilization by two haploid sperm ( 66\%) or fertilization by a single diploid sperm $(\sim 24 \%)$. Tetraploidy $(4 n=92)$ is uncommon, with embryos rarely surviving beyond 2 to 3 weeks of embryonic life.

Monosomy X accounts for 15 to $20 \%$ of chromosomally abnormal abortuses and is lethal in 95 to $98 \%$ of conceptions. Monosomy X abortuses usually consist of only an umbilical cord stump. If survival persists until later in gestation, anomalies characteristic of Turner syndrome may be seen like cystic hygroma, generalized edema, cardiac defects. Although wellrecognized clinically, 45, X liveborns are rare.

Autosomal monosomy is lethal prior to or just beyond implantation. No nonmosaic cases have survived to clinical recognition.

\section{Relationship between Recurrent Losses and Numerical Chromosomal Abnormalities}

In both preimplantation and first-trimester abortions, recurrent aneuploidy occurs more often than expected by chance. ${ }^{33}$ Recurrent aneuploidy is a frequent explanation for recurrent pregnancy losses, at least until the number of losses reaches or exceeds four. In a given family successive abortuses are thus likely to be either recurrently normal or recurrently abnormal (Table 2). That is, if the complement of the first abortus is abnormal, the likelihood is increased that the complement of the second abortus will also be abnormal. ${ }^{34}$ Recurrence usually involves trisomy, although not necessarily for the same chromosome. ${ }^{35}$ Observed the same phenomenon in couples undergoing repeated PGD, who were studied for monogenic indications, not an age-related phenomenon.

\section{PGD for Recurrent Pregnancy Losses}

Given the above, the rationale for performing PGD aneuploidy testing to transfer only euploid embryos would seem unassailable. Because the rationale is applicable only for couples experiencing recurrent aneuploidy, however at least one loss should ideally have been documented as aneuploid. If no information is available, one can perform FISH or array CGH on archived specimens embedded in paraffin. If this is not possible, one should acknowledge that half of recurrent pregnancy losses will not have been due to recurrent aneuploidy.

Randomized clinical trials (RCTs) have not been performed for this indication alone, but PGD seems beneficial in reducing further abortions and cumulatively resulting in livebirths. ${ }^{13,37-39}$ An excellent surrogate involves comparison to objective criteria, using the Brigham formula, ${ }^{40}$ which takes into account maternal age and the number of prior abortions to derive the likelihood of a pregnancy loss. Munné et al. ${ }^{13}$ observed losses in only $13 \%$ of couples, who underwent PGD, compared to an expected rate (Brigham) of 33\%. Benefit was predictably greatest for women older than 35 (39\% expected vs 13\% observed; $P<0.001)$.

PGD is best for aneuploidy testing undertaken in infertile couples having 6 to 8 morphologically "high grade" day 3 embryos. This offers reasonable likelihood of finding 1 to 2 euploid embryos to transfer. ${ }^{1}$

\section{Improving ART Pregnancy Rates (Absent Other Indications)}

PGD aneuploidy testing has been applied solely to improve pregnancy rates in infertile women, who require ART for infertility but are not at increased risk for genetic abnormalities in their offspring. Again the rationale is presumed benefit of transferring euploid embryos only. Indeed, the well-known decline in ART pregnancy rates beginning late in the fourth decade results from high embryonic loss due to aneuploidy. This is evident from undiminished ART pregnancy rates when transfer of donor embryos or donor oocytes are used in women in their fifth decade. In the United States, the overall 'take-

Table 2: Recurrent aneuploidy: relationship between karyotypes of successive abortuses

\begin{tabular}{|c|c|c|c|c|c|c|}
\hline \multirow{2}{*}{$\begin{array}{l}\text { Complement } \\
\text { of first abortus }\end{array}$} & \multicolumn{5}{|c|}{ Complement of second abortuses ${ }^{33}$} & \multirow[b]{2}{*}{$\begin{array}{l}\text { De novo } \\
\text { rearrangement }\end{array}$} \\
\hline & Normal & Trisomy & Monosomy & Triploidy & Tetraploidy & \\
\hline Normal & 142 & 18 & 5 & 7 & 3 & 2 \\
\hline Trisomy & 31 & 30 & 1 & 4 & 3 & 1 \\
\hline Monosomy X & 7 & 5 & 3 & 3 & 0 & 0 \\
\hline Triploidy & 7 & 4 & 1 & 4 & 0 & 0 \\
\hline Tetraploidy & 3 & 1 & 0 & 2 & 0 & 0 \\
\hline $\begin{array}{l}\text { De novo } \\
\text { rearrangement }\end{array}$ & & & & & & \\
\hline rearrangement & 1 & 3 & 0 & 0 & 0 & 0 \\
\hline
\end{tabular}

Data from Warburton ${ }^{33}$ 
home baby rate’ for non-donor ART (deliveries per retrieval) was $31 \%$ in cycles begun in 2001. However, rates were $38.9 \%$ for women $<35$ years old, 32.9\% for age 35 to 37, 24.3\% for age 38 to 40 and $11.1 \%$ for age $>40$ years. ${ }^{41}$ Paralleling the increasing aneuploidy rates with increasing maternal age is an increasing miscarriage rate. Thus, the strategy of transferring euploid embryos is obvious.

\section{Favorable Descriptive Reports}

Favorable results were reported from experienced centers worldwide beginning in the late 1990s. ${ }^{36-39,42-44}$ Salutary results were also evident, when comparisons were made to age-matched women not undergoing PGD. Two smaller RCTs conducted in the United States ${ }^{45,46}$ showed improved pregnancy rates, although neither was sufficiently powered. Many centers in the United States and Europe thus offered PGD to improve pregnancy rates in older women, although none of the largest centers have been able to complete an RCT.

\section{Randomized Clinical Trials and Their Arguable Results}

Given inability to conduct RCTs in more experienced centers, less experienced or perhaps even still evolving centers conducted RCTs. In some centers this was apparently done prior to clinically offering PGD aneuploidy testing. Thus, embryological biopsy skills were still being gained. None of these studies showed significant improvement in pregnancy rates. ${ }^{46-52}$ At least one ${ }^{49}$ showed harmful effect, whereas results in others were inconclusive or showed no benefits at least of significance. Conclusions have been widely criticized by ourselves and others ${ }^{53-56}$ on grounds of questionable technical process, diagnostic uncertainty and arguable indications.

Given the yet unresolved controversy, it is worth considering in details, the two most widely cited studies. The first was an RCT conducted in a premier Belgian ART center. ${ }^{47}$ Women aged 36 to 39 years, who were randomized to PGD had a clinical pregnancy rate ( $>12$ weeks) of $16.5 \%$ per embryo vs $10.4 \%$ in controls $(P=0.06)$. However, the take-home baby rate was not significantly different. The major pitfall in this RCT was removal of two blastomeres. Loss of even a single blastomere diminishes embryo viability by approximately $10 \%$. Loss of two cells diminishes viability by $40 \% .^{53}$ Indeed, this Belgian center reported in a later study the deleterious effects of removing two rather than one cell. ${ }^{57}$ Reported live birth rates were 37.4 and $22.4 \%$ after removal of one versus two cells, respectively. The authors acknowledged that the criticism of Cohen et $\mathrm{al}^{53}$ and others concerning removal of two cells 'seems justified ${ }^{57}$.

In a second well publicized RCT, ${ }^{49}$ there were several problems. Foremost was that in $20 \%$ of blastomeres, there were no diagnostic results. This percentage is much higher than expected. ${ }^{58,60}$ The high "no result" rate could have reflected embryo damage or, less likely, diagnostic inexperience. Irrespective, this problem was confounded by the low mean total number of embryos (4.8/cycle). A minimum of 6 morphologically normal embryos is recommended for proceeding with PGD. ${ }^{56,58} \mathrm{~A}$ third problem was the method of statistical analysis. When PGD was successful (defined as seven chromosomes tested and at least one euploid embryo transferred), the pregnancy rate was $16.8 \%$ per embryo. When no biopsy was performed (true control), the pregnancy rate was $14.7 \%$ per embryo, or $13 \%$ lower than with PGD. However, there was a (unintended) third group, given the many cases in which biopsy was performed but no diagnostic result achieved. The pregnancy rate was $6 \%$ in this group. The authors then applied intent-to-treat statistical analysis, a method dictating that all cases must remain assigned to their original group even if they do not complete their assigned technical protocol (i.e. PGD). Although there is theoretical validity in adhering to this design in straightforward pharmaceutical trials, the logic of doing so, when the randomization involves a (failed) surgical procedure (i.e. biopsy and diagnosis) seems highly arguable. Nonetheless rigidly applying this statistical method, the true PGD group (16.8\% pregnancy per embryo) was pooled with the de facto sham group (6\%) to yield a blended 'PGD' live birth rate of $24 \%$ per cycle, which compared unfavorably to the statistically higher 35\% non-biopsied, non-PGD group.

In conclusion, no robust RCT has in our opinion been conducted in an experienced center adhering to appropriate indications, having biopsy expertise, studying only woman with the requisite number of embryos and having diagnostic experience. Such a study is planned by the ESHRE PGD consortium, using polar bodies and array CGH, and will be discussed below. ${ }^{59}$

\section{Changing Attitudes toward Source of Embryonic DNA}

There are three potential sources of embryonic DNA:

1. Polar body from the unfertilized (meiosis I) or just fertilized (meiosis II) oocytes.

2. Blastomere, from the 3rd day six to eight-cell cleavage stage embryos.

3. Trophectoderm from the 5th to 6th day blastocyst. See Verlinsky and Kuliev ${ }^{61}$ for details on these biopsy techniques.

Blastomere biopsy has traditionally been the most widely used source for preimplantation embryonic DNA. Zona pellucida surrounding the embryo can be traversed by mechanical, laser or chemical means in order to extract a cell(s) and, hence a nucleus. The first two methods are used most commonly. Most centers remove one cell because, as noted above, removing only one cell is believed to minimize decrease in embryo survival (i.e. only $10 \%$ reduction). ${ }^{53}$ This $10 \%$ figure was deduced on the basis of numbers of blastomeres surviving following thawing cryopreserved embryos. Extrapolating loss of viability (pregnancy rates) to biopsied embryos not subjected to cryopreservation may or may not be totally applicable. However, directly comparing PGD results after the removal of 
one vs two blastomeres shows lower rates in the latter. Thus, any argument revolves not around the principle that removing two cells are more deleterious than one, but rather the magnitude of the deleterious effect. ${ }^{57}$ A $40 \%$ reduction in pregnancy rate associated with loss of two blastomeres naturally casts aspersions on protocols that routinely remove two blastomeres. A further pitfall of blastomere biopsy is that the cell may be unrepresentative. The single blastomere subjected to PGD could be the unlikely product of a single, unique mitotic nondisjunctional event and, hence unrepresentative of the other 7 blastomere (embryo). All other cells could be normal. That the number of monosomic blastomeres exceeds the number of trisomic blastomeres suggests anaphase lag. Irrespective, in either case an opportunity for transfer of a normal embryo may be lost.

Trophectoderm-biopsy of the trophectoderm of the 5th to 6th day, 120-cell blastocyst is a second approach. More cells can be removed at this stage, potentially facilitating diagnosis. Trophectoderm forms the placenta. Thus, embryonic cells per se are not removed. Trophectoderm biopsy is generating increased attention given the recently recognized value of permitting 5 days in vitro culture prior to transfer. The additional 2 to 3 days in culture allows self-selection against non-thriving embryos. One-third of embryos with chromosomal abnormalities being are selected against between days 3 and 5 . However, PGD is still necessary to exclude the remaining aneuploidies. Investigators have demonstrated feasibility of

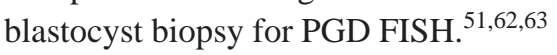

Polar body - this method is technically more difficult, but belatedly has been recognized as the most reliable source of preimplantation embryo DNA for cytogenetic analysis. Haploid chromosomal status of the oocyte can be deduced following analysis of the first and/or second polar biopsy. ${ }^{42}$ The underlying principle is that the chromosomal complement of the first polar body is complementary to that of the primary oocyte. Oocytes having a euploid polar body are deduced by complementation to be genetically normal and allowed to be fertilized in vitro, the resulting embryo transferred for potential implantation. Conversely, a monosomic polar body indicates a trisomic oocyte. Thus, fertilization would not be allowed to proceed. That is, if the first polar body failed to show the expected single chromosome 21, the oocyte would be presumed to have 21 chromosomes and, hence generate a trisomic zygote once fertilized by a (normal) haploid sperm.

Biopsy of the first polar body can uniquely provide preconceptional information. This becomes the only alternative, if one legally must limit the number of oocytes that can be fertilized or embryos transferred. Biopsy of the first polar body even allows, in the absence of recombination, euploid oocytes to be identified prior to conception. If restrictive legislation exists, only euploid oocytes can be chosen to be fertilized, ${ }^{64}$ if the second polar body is not biopsied.

Polar body biopsy may need to be followed by sequential testing of blastomere or blastocyst, either to exclude rarer paternally derived abnormalities or to clarify certain cases in which diagnostic results are incomplete. ${ }^{65,66}$ Blastomeres or trophectoderm biopsy is then required, and desirable routinely to exclude sex chromosome aneuploidy. However, biopsy of polar bodies followed later by biopsy of the embryo does not seem to decrease pregnancy rates compared to either alone. ${ }^{67}$

Centers technically experienced in polar body biopsy appear to have 25 to $30 \%$ pregnancy rates, comparable to those achieved using blastomere biopsy. No attempts have been made to determine relative safety of blastomere $v s$ polar body biopsy, reflecting until recently the reality that few centers performed polar body biopsy.

\section{Increasing Accuracy in Predicting Euploid Embryos (24 Chromosomes Analysis)}

Preimplantation cytogenetic analysis (numerical or structural) has historically depended on FISH using chromosome specific probes. ${ }^{68-71}$ Obtaining accurate results requires an intact nucleus, which in turn requires facile biopsy technique. The most informative results are achieved, when removal of a blastomere or polar body can be accomplished in 3 minutes or less. If the embryo or gametes requires longer to biopsy the cell (and embryo) may be damaged as result of desiccation, temperature changes or alterations in osmolarity. The high error rates and high numbers of noninformative "no results" embryos in certain RCTs probably reflect embryo damage. ${ }^{56,72,73}$

As discussed previously, approximately 50 to $72 \%$ of aneuploidy can be detected using 9 chromosomes with an increase in detection of 80 to $91 \%$, if 12 chromosomes are interrogated (e.g. X, Y, 8, 13, 14, 15, 16, 17, 18, 20, 21, 22). ${ }^{31,74-80}$ Interrogation of these chromosomes can be accomplished over 2 to 3 hybridization cycles, with a good quality embryonic cell. When a given probe fails to yield results for a given chromosome, retesting with a "rescue probe" for a different loci on the same chromosome can successfully lower the 'no result' rate. ${ }^{81}$

Two approaches are being pursued to obtain information on all 24 chromosomes. The first involves FISH hybridization covering all 24 chromosomes. Using single blood cells, AurichCosta et $\mathrm{al}^{82}$ enumerated 24 chromosomes on a single slide, performing 6 successive oligo-FISH cycles each with 4 chromosomes. Griffin et $\mathrm{al}^{83}$ achieved similar results successfully on blastomeres utilizing a commercially available 24-color FISH assay, with four hybridization rounds being accomplished within 24 hours. As alluded to above in the context of addressing couples with pregnancy losses, Munné et $\mathrm{al}^{74}$ selected only 10 to 12 chromosomes, but differentially choose on the basis of relative maternal age (e.g. chromosomes 2, 4, 7 in younger women, chromosome 11 in older women). Given that rarer trisomies are so often found concomitantly with another trisomy (double trisomy), screening for only the 10 allowed 89\% (382/427) of embryos to be classified as either normal or abnormal testing 12 chromosomes classified 91\% $(389 / 427) .^{74}$ 
The second general approach is a genome-wide molecular approach, using either CGH or SNPs. The approach could involve individually identified SNP analysis or a commercial available SNP platform (array). The latter is termed whole genome arrays. The array need not be the 600,000 SNPs array often used in genome works and postnatal testing. In fact, some information may wish to be "masked" in order to avoid unwanted adventitious information. Alternatively, lower resolution Bacterial Artificial Chromosome (BAC) arrays can provide a sufficient number of SNPs to exclude aneuploidy for each chromosome. Irrespective of approach, the number of chromosomes in a single cell (2 or 3) can be deduced on the basis of transmission of parental SNPs to the embryo. Altered allele number (2 vs 3 ) or altered allele ratio (2:1 vs 1:1) predicts presence or absence of trisomy respectively. Single cell testing for genome wide SNPs was accomplished reliably on a single blastomere by Handyside et al..$^{84}$ Vanneste et al. ${ }^{85}$ and Johnson et al. ${ }^{86}$ Analysis can be rapid enough for normal embryos to be transferred in the same cycle. Using SNPs, Treff et al. ${ }^{87}$ accomplished this in $4 \mathrm{~h}$.

Another approach is comparative genome hybridizations (CGH). The principle involves comparing the relative amount of genomic DNA is an unknown test case (e.g. embryo) with that of known normal genotype. Additional DNA from the test case would indicate trisomy. Metaphase CGH was first reported a decade ago. ${ }^{88-90}$ Now, Wells et $\mathrm{al}^{91,92}$ have used metaphase (CGH) to test all 24 chromosomes. In one small series, over $90 \%$ of blastocysts gave informative results, and 36 of 42 cycles (86\%) resulted in clinical pregnancy, non-PGD blastocysts showed a lower $60 \%$ pregnancy rate.

Array CGH in PGD is being profitably applied in conjunction with vitrification, a newly developed method of cryopreservation. Vitrification allows less harried array CGH analysis by SNPs or CNV, followed by embryo transfer at a later date, if normal. Using array CGH (Illumina platform), Wells ${ }^{93}$ reported that the probability of an individual embryo achieving a pregnancy was $66.7 \%$, vs $27.9 \%$ without PGD. Sher et al. ${ }^{94}$ reported a remarkable increase in birth rates transferring blastocysts which as 3rd day embryos underwent biopsy, CGH, followed by vitrification and then transfer upon thawing at a later time: $48 \%$ birth rate (45/94) per transferred CGH-tested blastocyst vs only 15\% (57/382) in non-CGHtested blastocysts.

Application of high-resolution molecular cytogenetics may soon shed further light on the parental contributions to aneuploidy. This was highlighted in the recent report of single cell PGD utilizing genome wide single nucleotide polymorphism (SNP) arrays. ${ }^{84}$ The advantage of these techniques, sometimes called 'karyomapping', is that they allow concomitant Mendelian analysis of genotypes, through analysis of inheritance of the four parental haplotypes and the position of any recombination event. If such technologies were routinely adopted in IVF clinics, preimplantation would extend beyond merely aneuploidy to detailed information on specific genes or parts of chromosomes. Information could be provided on certain monogenic traits and uniparental disomy (e.g. BeckwithWiedemann syndrome) of relevance to ART. The introduction of SNP array technology for PGD would also provide a wealth of data to further our understanding of recombination and its parental origin. This will in turn generate information on chromosomal and recombination errors that can be applied to infertile couples.

\section{Current Status of PGD Aneuploidy Testing to Improve ART Pregnancy Rates}

The ESHRE PGD Consortium has generated a position statement recommending that PGD aneuploidy testing should interrogate embryonic cells other than blastomeres. Further, ESHRE has stated that PGD based on chromosomal status of the oocyte, deduced from its complementary first or second polar bodies, paradoxically appears to be a more reliable indication of embryo status itself. The problem of mitotic nondisjunction in cleavage stage embryo is obviated. ESHRE has also recommended that diagnostic methods other than FISH alone be used, ${ }^{95}$ namely array CGH. Given these advances, an RCT is now planned to determine whether the use of these approaches improve liveborn ART pregnancy rates or not. A recent study is conducted to examine the robustness of polar body (PB) 1 and 2 in predicting oocyte and, hence embryo status has been conducted. Two centers experienced in PB biopsy were utilized. PB biopsy analysis performed at one center was compared to oocyte analysis at the other and vice versa. Concordance between PB 1 and 2 and oocyte was $89 \% .{ }^{59}$

Pending results of the ESHRE PGD consortium trial, the following seem appropriate counsel for women seeking to improve their likelihood of ART success.

1. Apply PGD only for women of maternal age, perhaps $>37$ years old.

2. Continue to pursue PGD in a given cycle only, if there are six to eight morphologically normal embryos, two to three genetically normal embryos can thus be reasonably expected. Given fewer embryos, PGD probably should not be pursued to help a couple reach resolution in the case of repeated ART failures.

3. Use only highly skilled embryologists.

4. Interrogate at least 8 and preferably 10 to 12 chromosomes by FISH, or preferably all 24 chromosomes by FISH or array/metaphase CGH.

\section{REFERENCES}

1. Simpson JL. Preimplantation genetic diagnosis at 20 years. Prenat Diagn 2010;30:682-95.

2. Simpson JL. Early Pregnancy Loss. In: Kovacs G (Ed). The subfertility handbook: A Clinician's guide (2nd ed). London: Cambridge University Press, in press.

3. Egozcue J, Blanco J, Anton E, Egozcue S, Sarrate Z, Vidal F. Genetic analysis of sperm and implications of severe male infertility: A review. Placenta 2003;24:S62-S65. 
4. Simpson JL, Meters CM, Martin AO, Elias S, Ober C. Translocations are infrequent among couples having repeated spontaneous abortions but no other abnormal pregnancies. Fertil Steril 1989;51:811-14.

5. Simpson JL, Otano L. Prenatal genetic diagnosis. in: Gabbe SA, Niebyl JF, Simpson JL (Eds). Obstetrics: Normal and problem pregnancies (5th ed). New York: Churchill-Livingstone 2007;152-83.

6. Daniel A, Hook EB, Wulf G. Risks of unbalanced progeny at amniocentesis to carriers of chromosome rearrangements: Data from United States and Canadian laboratories. Am J Med Genet 1989;33:14-53.

7. Tempest HG. Meiotic recombination errors, the origin of sperm aneuploidy and clinical recommendations: A review. Syst Biol Reprod Med 2010;56:1-11.

8. Martin RH. Cytogenetic determinants of male fertility. Hum Reprod Update 2008;14:379-90.

9. Conn CM, Harper JC, Winston RM, Delhanty JD. Infertile couples with Robertsonian translocations: Preimplantation genetic analysis of embryos reveals chaotic cleavage divisions. Hum Genet 1998;102:117-23.

10. Conn CM, Cozzi J, Harper JC, Winston RM, Delhanty JD. Preimplantation genetic diagnosis for couples at high-risk of Down syndrome pregnancy owing to parental translocation or mosaicism. J Med Genet 1999;36:45-50.

11. Gianaroli L, Magli MC, Ferraretti AP, Munné S, Balicchia B, Escudero T, et al. Possible interchromosomal effect in embryos generated by gametes from translocation carriers. Hum Reprod 2002;17:3201-07.

12. Scriven PN, Flinter FA, Braude PR, Ogilvie CM. Robertsonian translocations:Reproductive risks and indications for preimplantation genetic diagnosis. Hum Reprod 2001;16:226773.

13. Munné S, Escudero T, Fisher J, Chen S, Hill J, Stelling JR, et al. A negligible interchromosomal effect in embryos of Robertsonian translocation carriers. Reprod Biomed Online 2005;10:363-69.

14. Goddijn M, Joosten JHK, Knegt AC, Vander, et al. Clinical relevance of diagnosing structural chromosome abnormalities in couples with repeated miscarriage. Hum Reprod 2004;19:1013-17.

15. Sugiura-Ogasawara M, Ozaki Y, Sata T, Suzumori N, Suzumori K. Poor prognosis of recurrent aborters with either maternal or paternal reciprocal translocation. Fertil Steril 2004;81:367-73.

16. Stephenson MD, Sierra S. Reproductive outcomes in recurrent pregnancy loss associated with a parental carrier of a structural chromosome rearrangement. Hum Reprod 2006;21:1076-82.

17. Fritz MA, Schattman G. Reply of the Committee: Parental translocations and need for preimplantation genetic diagnosis? Distorting effects of ascertainment bias and information need for rich families. Fertil Steril 2008;90:892-93.

18. Otani T, Roche M, Mizuike M, Colls P, Escudero T, Munné S. Preimplantation genetic diagnosis significantly improves the pregnancy outcome of translocation carriers with a history of recurrent miscarriage and unsuccessful pregnancies. Reprod Biomed Online 2006;13:879-94.

19. Munné S, Morrison L, Fung J, Marquez C, Weier HUG, Bach $\mathrm{M}$, et al. Spontaneous abortions are reduced after preconception diagnosis of translocations. J Assist Reprod Genet 1998;15:29096.

20. Munné S, Sandalinas M, Escudero T, Fung J, Gianaroli L, Cohen J. Outcome of preimplantation genetic diagnosis of translocation. Fertil Steril 2000;73:1209-18.
21. Munné S, Fung J, Cassel C, Marquez C, Weier HUG. Preimplantation genetic analysis of translocations case-specific probes for interphase cell analysis. Hum Genet 1998;102:66374.

22. Verlinsky Y, Evsikov S. A simplified and efficient method for obtaining metaphase chromosomes from individual human blastomeres. Fertil Steril 1999;72:1127-33.

23. Verlinsky Y, Cieslak J, Evsikov S, Galat V, Kuliev A. Nuclear transfer for full karyotyping and preimplantation diagnosis for translocations. Reprod BioMed Online 2002;5:300-05.

24. Willadsen S, Levron J, Munné S, Schimmel T, Marchez C, Scott $\mathrm{R}$, et al. Rapid visualization of metaphase chromosomes in single human blastomeres after fusion with invitro matured bovine eggs. Hum. Reprod 1999;14:470-74.

25. Kuliev A, Janzen JC, Zlatopolsky Z, Kirillova I, Ilkevitch Y, Verlinsky Y. Conversion and nonconversion approach to preimplantation diagnosis for chromosomal rearrangements in 475 cycles. Reprod Biomed Online 2010;21:93-99.

26. Shkumatov A, Kuznyetsov V, Cieslak J, Ilkevitch Verlinsky Y. Obtaining metaphase spreads from single blastomeres for PGD of chromosomal rearrangements. Reprod Biomed Online 2007;14:498-503.

27. Traversa M, Leigh D. The application of a molecular strategy using STR for routine PGD in both reciprocal and Robertsonian translocation carriers. Ninth international conference on preimplantation genetics, Miami, Florida, April 23 to 25. Reprod Biomed Online 2009;18:S10.

28. Munné S, Alikani M. Tomkin G, Gripo J, Cohen J. Embryo morphology, development rates, and maternal are correlated with chromosome abnormalities. Fertil Steril 1995;64:382.

29. Pfeffer J, Pang MG, Hoegerman SF, Osgood CJ, Stacey MW, Mayer J, et al. Aneuploidy frequencies in semen fractions from ten oligoasthenoteratozoospermic patients donating sperm for intracytoplasmic sperm injection. Fertil Steril 1999;72(3):47278.

30. Rosebusch B. The incidence of aneuploidy in human oocytes assessed by conventional cytogenetic analysis. Hereditas 2004;141:97-105.

31. Colls P, Goodall N, Zheng X, Munné S. Increased efficiency of preimplantation genetic diagnosis for aneuploidy by testing 12 chromosomes. Reprod Biomed Online Oct 2009;19:532-38.

32. Munné S, Fragouli E, Colls P, Katz-Jaffe M, Schoolcraft W, Wells D. Improved detection of aneuploid blastocysts using a new 12 chromosomes FISH test. Reprod Biomed Online 2010;20:92-97.

33. Warburton D, Kliner JH, Stein Z, Hutzler M, Chin A, Hassold T. Does the karyotype of a spontaneous abortion predict the karyotype of a subsequent abortion? Evidence from 273 women with two karyotyped spontaneous abortions. Am J Hum Genet 1987;41:465-83.

34. Warburton D, Dallaire L, Thangavelu M, Ross L, Levin B, Kline J. Trisomy recurrence: A reconsideration based on North American data. Am J, Hum Genet 2004;75:376-85.

35. Rubio C, Simon C, Vidal F, Rodrigo L, Pehlivan T, Remohi J, Pellicer A. Chromosomal abnormalities and embryo development in recurrent miscarriage couples. Hum Reprod 2003;18:182-88.

36. Gianaroli L, Magli MC, Ferraretti AP, Munné S. Preimplantation diagnosis for aneuploidies in patients undergoing in vitro fertilization with poor prognosis: Identification of the categories to which it should be proposed. Fertil Steril 1999;72:837-44.

37. Munné S, Magli C, Cohen J, Morton P, Sadowy S, Gianaroli L, et al. Positive outcome after preimplantation diagnosis of aneuploidy in human embryos. Hum Reprod 1999;14:2191-99. 
38. Verlinsky Y, Tur-Kaspa I, Cieslak J, Bernal A, Morris R, Taranissi M, et al. Preimplantation testing for chromosomal disorders improves reproductive outcome of poor-prognosis patients. Reprod Biomed Online 2005;11:219-25.

39. Verlinsky Y, Kuliev A. PGD and its role in ART. In: Brinsden P (Ed). Textbook of IVF and assisted reproduction (3rd ed), London (UK): Parthenon publishing group 2005.

40. Brigham SA, Conlon C, Farquharson RG. A longitudinal study of pregnancy outcome following idiopathic recurrent miscarriage. Hum Reprod 1999;14:2868-71.

41. Society for assisted reproductive technology and the American society for reproductive medicine. Assisted reproductive technology in the United States: 2001 results generated from the American society for reproductive medicine/society for assisted reproductive technology registry. Fertil Steril 2007;87:1253-66.

42. Verlinsky Y, Kuliev A. Practical preimplantation genetic diagnosis, London (UK); Springer-Verlag 2005.

43. Gianaroli L, Magli MC, Ferraretti AP, Tabanelli C, Trengia V, et al. The beneficial effects of PGD for aneuploidy support extensive clinical application. Reprod BioMed Online 2005;10:633-40.

44. Munné S, Sandalinas M, Escudero T, Velilla E, Walmsley R, Sadowy S, et al. Improved implantation after preimplantation genetic diagnosis of aneuploidy. Reprod Biomed Online 2003;7:91-97.

45. Werlin L, Rodi I, DeCherney A, Marello E, Hill D, Munné S. Preimplantation genetic diagnosis as both a therapeutic and diagnostic tool in assisted reproductive technology. Fertil Steril 2003;80:467-68.

46. Mersereau J, Pergament E, Zhang X, Milad MP. Preimplantation genetic screening to improve in vitro fertilization pregnancy rates: A prospective randomized controlled trial. Fertil Steril 2008;90:1287-89.

47. Staessen C, Platteau P, Van Assche E, Michiels A, Tournaye H, Camus $\mathrm{M}$, et al. Comparison of blastocyst transfer with or without preimplantation genetic diagnosis for aneuploidy screening in couples with advanced maternal age: A prospective randomized controlled trial. Hum Reprod 2004;19:2849-58.

48. Staessen C, Verpoest W, Donoso P, Haentjens P, Van der Elst $\mathrm{J}$, Liebaers I, et al. Preimplantation genetic diagnosis does not improve delivery rate in women under the age of 36 following single-embryo transfer. Hum Reprod 2008;23:2818-25.

49. Mastenbroek S, Twisk M, van Echten-Arends J, SikkenaRaddatz Bm Korevaar JC, Verhoeve HR, et al. In vitro fertilization with preimplantation genetic screening. N Engl J Med 2007;357:9-17.

50. Hardarson T, Hanson C, Lundin K, Hillensjo T, Nilsson L, Stevic J, et al. Preimplantation genetic screening in women of advanced maternal age caused a decrease in clinical pregnancy rate: A randomized controlled trial. Hum Reprod 2008;23:2617-21.

51. Schoolcraft WB, Fragouli E, Stevens J, Munne S, Katz-Jaffe MG, Wells D. Clinical application of comprehensive chromosomal screening at the blastocyst stage. Fertil Steril Nov. 23, 2009 (Epub ahead of print).

52. Debrock S, Melotte C, Spiessens C, Peeraer K, Vanneste E, Meeuwis L, et al. Preimplantation genetic screening for aneuploidy of embryos after in vitro fertilization in women aged at least 35 years: A prospective randomized trial. Fertil Steril 2010;93:364-73.

53. Cohen J, Grifo J. Multicenter trial of preimplantation genetic screening reported in the New England Journal of Medicine: An indepth look at the findings. Reprod Biomed Online 2007;15:305-66.
54. Munné S, Cohen J, Simpson JL. In vitro fertilization with preimplantation genetic screening. N Engl J Med 2007;25: 1769-70.

55. Munné S, Gianaroli L, Tur-Kaspa I, Magli C, Sandalinas M, et al. Substandard application of preimplantation genetic screening may interfere with its clinical success. Fertil Steril 2007;88:781-84.

56. Simpson JL. What next for preimplantation genetic screening? Randomized clinical trial in assessing PGS: Necessary but not sufficient. Hum Reprod 2008;23:2179-81.

57. DeVos A, Staessen C, De Rycke M, Verpoest W, Haentjens, et al. Impact of cleavage stage embryo biopsy in view of PGD on human blastocyst implantation: A prospective cohort of single embryo transfers. Hum Reprod 2009;24:2988-96.

58. Preimplantation genetic diagnosis international society (PGDIS). Guidelines for good practice in PGD: Program requirements and laboratory quality assurance. Reprod Biomed Online 2008;16:134-47.

59. Geraedts J. Results from the polar body proof of principle study. 26th annual meeting of the European society of human reproduction and embryology, Rome, Italy, June 27 to 302010. Hum Reprod 2010;25:42.

60. Gutierrez-Mateo C, Sanchez Garcia J, Fischer J, Tormasi S, Cohen J, Munné S, et al. Preimplantation genetic diagnosis of single gene disorders: Experience with more than 200 cycles conducted by a reference laboratory in the United States. Feril Steril 2009;92:1544-56.

61. Verlinsky Y, Kuliev A. Atlas of preimplantation genetic diagnosis (2nd ed). Boca Raton (FL): Taylor and Francis; 2005.

62. McArthur SJ, Leigh D, Marshall JT, De Boer KA, Jansen RPS. Pregnancies and live births following biopsy and PGD analysis of human embryos at the blastocyst stage. Fertil Steril 2005;84:1628-36.

63. McArthur SJ, Leigh D, Marshall JT, Gee AJ, De Boer KA, Jansen RP. Blastocyst trophectoderm biopsy and preimplantation genetic diagnosis for familial monogenic disorders and chromosomal translocations. Prenat Diagn 2008;28:434-42.

64. Gianaroli L, Magli MC, Lappi M, Capoti A, Robles F, Ferraretti AP. Preconception diagnosis. 9th international conference on preimplantation genetics, Miami, Florida, April 23 to 25. Reprod Biomed Online 2009;18:S5.

65. Kuliev A, Cieslak J, Verlinsky Y. Frequency and distribution of chromosomal abnormalities in human oocytes. Cytogenet Genome Res 2005;111:193-98.

66. Kuliev A, Verlinsky Y. Preimplantation genetic diagnosis: Technological advances to improve accuracy and range. Reprod Biomed Online 2007;16:532-38.

67. Cieslak J, Tur-Kaspa I, Ilkevitch Y, Bernal A, Morris R, Verlinsky Y. Multiple micromanipulations for preimplantation genetic diagnosis do not affect embryo development to the blastocyst stage. Fertil Steril 2006;85:1826-29.

68. Griffin DK, Handyside AH, Penketh RJ, Winston RM, Delhanty JD. Fluorescent in-situ hybridization to interphase nuclei of human preimplantation embryos with $\mathrm{X}$ and $\mathrm{Y}$ chromosome specific probes. Hum Reprod 1991;6:101-105.

69. Grifo JA, Boyle A, Tang YX, Ward DC. Preimplantation genetic diagnosis. In situ hybridization as a tool for analysis. Arch Pathol Lab Med 1992;116:393-97.

70. Grifo JA, Tang YX, Cohen J, Gilbert F, Sanyal M, Rosenwaks Z. Pregnancy after embryo biopsy and coamplification of DNA from X and Y chromosomes. JAMA 1992;268:727-29.

71. Munne S, Lee A, Rosenwaks Z, Grifo J, Cohen J. Diagnosis of major chromosome aneuploidies in human preimplantation embryos. Hum Reprod 1993;8:2185-92. 
72. Munné S, Cohen J, Simpson J. In vitro fertilization with preimplantation genetic screening. N Engl J Med 2007;25:176970.

73. Munné S, Fianaroli L, Tur-Kaspa I, Magli C, Sandalinas M, Gripo J, et al. Substandard application of preimplantation genetic screening may interfere with its clinical success. Fertil Steril 2007;88:781-84.

74. Munné S, Fragouli E, Colls P, Katz-Jaffe M, Schoolcraft W, Wells D. Improved detection of aneuploid blastocysts using a new 12 chromosome FISH test. Reprod Biomed Online 2010;20:92-97.

75. Jobanputra V, Sobrino A, Kinney A, Kline J, Warburton D. Multiplex interphase FISH as a screen for common aneuploidies in spontaneous abortions. Hum Reprod 2002;17:1166-70.

76. Gutierrez-Mateo C, Benet J, Wells D, Colls P, Bermudez MG, Sanchez-Garicia JF, et al. Aneuploidy study of human oocytes first polar body comparative genomic hybridization and metaphase II fluorescence in situ hybridization analysis. Hum Reprod 2004;19:2859-68.

77. Gutierrez-Mateo C, Benet J, Wells D, Sanchez-Garcia JF, Bermudez MG, Belil I, et al. Reliability of comparative genomic hybridization to detect chromosome abnormalities in first polar bodies and metaphase II oocytes. Hum Reprod 2004;19:211825.

78. Munné S, Bahce M, Sandalinas M, Escudero T, Marquez C, Velilla E, et al. Differences in chromosome susceptibility to aneuploidy and survival to first trimester. Reprod Biomed Online 2004;8:81-90.

79. Fragouli E, Wells D, Thornhill A, Serhal P, Faed MJ, Harper JC, et al. Comparative genomic hybridization analysis of human oocytes and polar bodies. Human Reprod 2006;21:2319-28.

80. Lathi RB, Westphal LM, Milki AA. Aneuploidy in the miscarriage of infertile women and the potential benefit of preimplantation genetic diagnosis. Fertil Steril 2007;89:353-357.

81. Colls P, Escudero T, Cekleniak N, Sadowy S, Cohen J, Munné S. Increased efficiency of preimplantation genetic diagnosis for infertility using "no result rescue". Fertil Steril 2007;88:53-61.

82. Aurich-Costa J, Ng C, Selvaggio S, Colls P, Bradley S. Oligonucleotide (ODN) fluorescence in situ hybridization (Oligo-FISH) and conventional FISH allow enumeration of 24 chromosomes in 6 successive hybridizations performed in a single day. Fertil Steril 2009;92:S50.

83. Griffin DK, Ioannou D, Gabriel AS, Tempest H, Grigorova M. Taylor J, et al. Novel perspectives on 24 chromosomes diagnosis in human preimplantation embryos. Chromosome Res 2009;17:554.

84. Handyside AH, Harton GL, Mariani B, Thornhill AR, Affara N, Shaw MA, et al. Karyomapping: A universal method for genome wide analysis of genetic disease based on mapping crossovers between parental haplotypes. J Med Genet 2010;PMID 19858130.

85. Vanneste E, Voet T, Le Caignec C, Ampe M. Konings P, Melotte $\mathrm{C}$, et al. Chromosome instability is common in human cleavagestage embryos. Nat Med 2009;15:577-83.

86. Johnson DS, Genelos G, Baner J, Ryan A, Cinnioglu C, Banjevic, et al. Preclinical validation of a microarray method for full molecular karyotyping of blastomeres in a 24 hours protocol. Hum Reprod 2010;25:1066-75.

87. Treff N, Tao X, Su J, Taylor D, Miller K, Scott R. Four hours 24 chromosomes aneuploidy screening using high throughput PCR SNP allele ratio analysis. Fertil Steril 2009;92:S49.

88. Voullaire L, Slater H, Williamson R, Wilton L. Chromosome analysis of blastomeres from human embryos by using comparative genomic hybridization. Hum Genet 2000;106:21017.

89. Wells D, Delhanty JD. Comprehensive chromosomal analysis of human preimplantation embryos using whole genome amplification and single cell comparative genomic hybridization. Mol Hum Reprod 2000;6:1055-62.

90. Wilton L, Williamson R, McBain J, Edgar D, Voullaire L. Birth of a healthy infant after preimplantation confirmation of euploidy by comparative genomic hybridization. N Engl J Med 2001;345:1537-41.

91. Wells D, Alfarawati S, Fragouli E. Use of comprehensive chromosomal screening for embryo assessment microarrays and CGH. Mol Hum Reprod 2008;14:703-10.

92. Wells D, Fragouli E, Alfarawaty S, Munne S, Schoolcraft WB, Katz-Jaffe MG. Highly significant improvement in embryo implantation and increased live birth rate achieved after comprehensive chromosomal screening: Implications for single embryo transfer. Fertil Steril 2009;92:S79.

93. Wells D, Fragouli E, Alfarawaty S, Schoolcraft WB, Munne S, Katz-Jaffe MG. Increased embryo implantation and high birth rates following comprehensive chromosomal screening of in vitro fertilized embryos. Reprod Biomed Online 2009;18:S10.

94. Sher G, Keskintepe L, Keskintepe M, Maassarani G, Tortoriello D, Brody S. Genetic analysis of human embryos by metaphase comparative genomic hybridization (mCGH) improves efficiency of IVF by increasing embryo implantation rate and reducing multiple pregnancies and spontaneous miscarriages. Fertil Steril 2009;92:1886-94.

95. Harper H, Coonen E, De Rycke M, Fiorentino F, Geraedts J, Goossens $\mathrm{V}$, et al. What next for preimplantation genetic screening (PGS)? A position statement from the ESHRE PGD consortium steering committee. Hum Reprod 2010;25:821-23.

96. Simpson JL. Jauniaux ERM. Pregnancy Loss In: Gabbe SA, Niebyl JF, Simpson JL (Eds). Obstetrics: Normal and problem pregnancies (5th ed). New York: Churchill-Livingstone 2007;628-49. 